\title{
Scripta
}

\section{Italia e la Corona d'Aragona: Dai tempi dei Borgia ai nostri tempi}

\author{
Italy and Crown of Aragon: From Borgia's age to now
}

\author{
Nancy De BenedetTo \\ nandeben@alice.it \\ Università di Bari - Aldo Moro
}

In questo monografico si è voluto offrire un panorama degli studi catalani che si svolgono in Italia partendo dalla segnalazione ragionata (Compagna-Puigdevall) dei più recenti contributi offerti dalla RISCAT, rivista dell'Associazione Italiana di Studi Catalani.

Si è anche inteso raccogliere inediti (Ripa), traduzioni (Siviero -quest'ultimo nella sezione di recensioni di questo numero di SCRIPTA) e interventi di ricerca letteraria prodotti da studiosi operativi in Italia (Concina, Bou, Rigobon, Turull) o in altri casi, dato l'interesse specifico centrato sulla reciprocità dei rapporti tra alcuni dei protagonisti del passato o del secolo scorso delle due aree geoculturali di interesse, a Barcellona (Gavagnin), Valencia (Roig) e Santander (Colella).

Si è voluto inoltre annunciare un romanzo di prossima pubblicazione che ritengo sia molto importante perché ci riporta alle origini della piccola tradizione novecentesca dei libri tradotti dal catalano. Si tratta di Solitudine di Caterina Albert, che uscirà a Roma per i tipi di Eliot, intorno alla metà del prossimo mese di giugno, su traduzione di Ursula Bedogni. L'unica traduzione del romazo era del 1918, l'aveva fatta Alfredo Giannini sulla base della terza edizione pubblicata a Barcellona dalla "Biblioteca Joventut", su concessione dell'autrice, per il benemerito editore Carabba di Lanciano nel 1918. Il testo si presentava, compito e molto rispettoso dell'integrità dell'originale, in due volumi, secondo le esigenze di manegevolezza che nelle prime due decadi del secolo scorso dovevano avere i libri di lettura che si volessero rivolgere ad un pubblico auspicabilmente crescente di lettori. Non ho studiato la fortuna di quella Solitudine, ma non l'ho mai trovata menzionata che in pochi studi specialistici; volentieri contribuirò almeno alla diffusione della nuova traduzione.

Si è pensato infine di ricordare dei libri, anche antologie di poesia (Siviero), e delle celebrazioni recenti dedicate all'eredità di maestri che hanno segnato le tappe salienti degli studi filologicoletterari in area romanza e classica, oltre che italo-catalana, tanto da coinvolgere diverse generazioni 
Nancy de Benedetto. Italia e la Corona d'Aragona: Dai tempi dei Borgia ai nostri tempi.

di studiosi, menzionati e in presentia. Ci riferiremo essenzialmente al Centenario Martí de Riquer e all'Omaggio a Carles Miralles organizzati da Giuseppe Grilli rispettivamente il 28 novembre 2014 e il 22 aprile 2015, presso il Dipartimento di Lingue, Culture e Letterature Straniere dell'Università Roma Tre.

La prima occasione di incontro, celebrata anche attraverso la ristampa di Cavalleria di Martí de Riquer (Roma, Aracne, 2014), è stato un omaggio della scuola romana di filologia romanza che, in quelle stesse date, non casualmente, si riuniva anche in ricordo di uno degli allievi prediletti di Riquer, da poco scomparso: Alberto Varvaro. Così l'intreccio riqueriano tra la storia e la letteratura è stato il leit motiv, declinato anche sotto forma di ricostruzione biografica, che ha unito i contributi dei filologi presenti, da Antonelli, a Bologna, a Antonucci, Beltrán, Lobera, Verlato, Giuseppina Brunetti, Isabel de Riquer. Per quanto riguarda la letteratura catalana, sono state tracciate sintesi molto efficaci dell'evoluzione degli studi aperti o significamente arricchiti dai contributi di Martí de Riquer, a cominciare da quello di Stefano Asperti che ha ricordato l'importanza della svolta rappresentata tra le scarse edizioni a stampa esistenti, dall'antologia dei poeti catalani del Trecento del 1953 sul percorso della storicizzazione della nascita della lingua che da questi giunge ad Ausiàs March.

Una grande fedeltà è stato invece il titolo con cui Giuseppe Grilli ha inquadrato gli studi riqueriani proprio sulle traduzioni castigliane di Ausiàs March, nel ruolo di fondatore, ultimo degli antichi, della poesia ispanica moderna. Grilli ha sottolineato come l'ordinamento per segnali della poesia marchiana, operata da Martì de Riquer abbia contribuito intuitivamente all'esaltazione dei valori civili della poesia catalana perché i segnali utilizzati come guida da Riquer sono stati innanzitutto quelli minori come Fulla morta; da qui, il salto è arduo, ma ben trovato, una tendenza indiscussa della poesia catalana al realismo, fino a poeti molto più recenti come Gabriel e Joan Ferrater. Dunque, procedendo ancora nella divisione per segnali, ha preso forma il canzoniere marchiano costituendo una composizione strutturale molto vicina a quello che sarebbe stato il sottogenere più ampiamente rappresentativo della poesia catalana novecentesca, dal Comte Arnau al Poema de la rosa als llavis, al Nabi, alle stesse Elegies di Bierville: il poemetto ellenistico.

Annamaria Annicchiarico ha infine parlato dello sviluppo della incontrovertibile conexió tra Corella e Martorell, individuata da Martí de Riquer sin dagli anni Quaranta, agli albori degli studi tirantiani e sedimentata sulle intuizioni riqueriane che avrebbero portato alla unicità autoriale del Tirant, alla evoluzione stilistica nel riuso delle fonti, alla ricostruzione dei presupposti di un progetto letterario che implica un reimpianto complessivo della finzione cavalleresca. I contributi che a partire da Riquer, si sono andati sedimentando in tradizione di studi, sono molti; si evita pertanto qui accuratamente di citarne alcuno perché la rapidità di questa introduzione non potrebbe che essere gravemente manchevole e arbitraria.

La seconda celebrazione che qui recensisco è stata un'occasione, commovente in diversi momenti, per ricordare la poliedrica, brillante figura di Carles Miralles, antichista, comparatista e poeta, fondatore dell'aula Carles Riba, che a sua volta ha lasciato un profondo segno negli ambiti di interesse 
a cui si è dedicato. Dal punto di vista della letteratura catalana, ancora una volta irrinunciabili sono risultati i contributi di Miralles nella ricostruzione dei miti che appaiono nella letteratura catalana contemporanea e in special modo ricordiamo La luce del dolore (Napoli, Liguoeri, 2009) e l'articolo sulla trasformazione dell'eroe tra «Epica e tragedia. Andata e ritorno» (in L'epica tra evocazione mitica e tragedia, Roma, Aracne, 2013).

Aggiungerei, per concludere questa breve rassegna di eventi e libri pubblicati recentemente, la squisita antologia di Poemes di Carles Miralles a cura di G. Grilli e E. Salibra (A \& B Editrice, Acireale, 2012). Si tratta di una piccola antologia di poesie tradotte in italiano con testo a fronte che presenta l'intero profilo di Miralles, ravvicinando efficacemente in apertura brevi testi in prosa in cui è contenuto il significato della poesia nella vita e nella vasta opera del poeta filologo. E' il percorso di una vita in cui la poesia nasce come ricerca di senso, sulle tracce di «una llengua a les palpentes, per enfotre'm dolgut del món que m’havia tocat i proclamar la meva esperança en el futur». La ricerca si spinge quindi in un percorso di conoscenza in cui il poeta non cerca più di dire quel che dovrebbe essere, ma di dare forma linguistica all'ipotesi del mistero del reale.

L'antologia si divide in due parti, la prima, a cura di Elena Salibra, in cui la scelta delle poesie, seppur esigua, riesce a tratteggiare il profilo diacronico del poeta, sin dal 1965, anno della prima raccolta di versi, La terra humida, dove la descrizione più esplicita, dei valori civili, oserei dire, va dal silenzio alla liberazione fulminante della denuncia del Manual de cosmologia (1969); dove l'ingiustizia è negli uomini che hanno dimenticato se stessi: Per tal d'oblidar/que sou mortals i que beu nascut / de mare, heu alçat banderes / de roba, fetes / de colors $i$ de vent, / davant de multituds / que us aclamen amb fervor, enfollides (p. 18). In questa fase, pur solo nelle pieghe della realtà assurda, roseggia una inevitabile, salvifica alba umanistica che sfocia in mari di luce e pace mattutina.

La seconda parte è costituita dai poemi di L'ombra dels dies roja, ultima raccolta di Miralles del 2009, dove ad uno sguardo maturo e compendiale dell'esperienza poetica e biografica dell'autore corrisponde un impeto giovanile e inaspettato, una energica «aggressione alla vita per trarne il succo goloso». Questo è secondo Grilli il senso 'paradossale' della raccolta, in cui ritroviamo un poeta "giovanissimo soprattutto per la radicalità della forma» (p. 36), sincero come Maragall, realista come Ferrater, elegante come Espriu, verace nella ricerca del principio umanistico della Risposta tanto crudele di Edipo: «Ignora uomo, le sfingi, / guarda di non incontrarne mai / nessuna lungi il tuo cammino / e fatti il segno della croce se di notte / senti che si parla di loro». / Ancora oggi l'opacità del cielo / certi giorni trattiene gli occhi degli uomini. / Tra l'uliveto e la vigna. (46) 